\title{
DETECTION OF SYMMETRIC JUNCTIONS IN BIOLOGICAL IMAGES USING 2-D STEERABLE WAVELET TRANSFORMS
}

\author{
Zsuzsanna Püspöki, Cédric Vonesch*and Michael Unser* \\ *Biomedical Imaging Group, École Polytechnique Fédérale de Lausanne (EPFL), Switzerland
}

\begin{abstract}
We present a method for designing steerable wavelets that can detect local centers of symmetry in images. Based on this design, we then propose an algorithm for estimating the locations and the orientations of M-fold symmetric junctions in biological micrographs.

The analysis with 2-D steerable wavelets allows us to have detections at different scales and arbitrary orientations. Owing to the steering property of our wavelets the detection is fast and accurate.

We provide experimental results on both synthetic images and biological micrographs to demonstrate the performance of the algorithm.
\end{abstract}

Index Terms - symmetries, steerability, wavelets

\section{INTRODUCTION}

Bilateral and 3-fold symmetric objects are common in nature. In particular, they can be found in various biological samples with hexagonal structures, such as the retinal pigment of the human eye, the parenchyma of maize, the surface of many diatoms and the stem cross-section of plants like convallaria. The ability to detect junction points with M-fold symmetry can thus be very useful for the quantitative analysis of microscopic images. Indeed, in addition to generating valuable image statistics, it can support further image-processing tasks such as cell segmentation. The challenge is to extract the key points accurately even when the image is corrupted with noise.

Most existing approaches to junction detection fall in one of the following categories: methods based on detecting and grouping edges [1], those relying on structural image analysis [2] and approaches based on matching parametric junction templates [3], including designs based on steerability $[4,5]$. Many detectors from the latter class are designed for specific types of junctions, and most (with the exception of steerable designs) involve discretized angles when matching the template to image features. The other classes primarily differentiate between junctions and edges or other keypoints, and do not always separate junctions of different multiplicities or symmetry orders.

In this paper, we propose an algorithm for junction detection in a scale and rotation invariant way. The core of our method is based on 2-D steerable wavelet frames, which provide both a multiscale analysis and angular selectivity. More specifically, we design 2-D steerable wavelets that are polar separable in the Fourier domain. The frequency localization of these wavelets is defined by a radial function, while their symmetry pattern is specified by an angular profile.

The approach presented here differs from existing methods in several ways. First of all, our framework has a multiresolu- tion detection scheme. In addition, since the analysis functions constitute a tight frame, they can be used for reconstructing the image. The Parseval-type identity for tight frames also allows for an energy-based analysis in the transform domain. The property of steerability, and our fast and accurate steering method based on polynomial root finding, make it possible to detect symmetry centers and junctions at arbitrary orientations without any discretization of the rotation angle. Finally, we use a variational framework for systematically deriving steerable templates with the desired symmetry properties and maximal angular concentration. As a result of this variational formulation, our detectors have optimal energy concentration with respect to the angular patterns of interest. The formulation is similar to Slepian filter design and can be recast as a low-dimensional eigenvector problem [6].

\section{GENERAL FRAMEWORK}

To introduce the notations, we recall the definition of steerability based on [7], and the parametric framework for 2-D steerable wavelet transforms described in [8]. The detector construction is discussed next.

\subsection{Steerability}

In this paper we use $x$ and $(r, \theta)$, and $\omega$ and $(\rho, \phi)$, for the Cartesian and polar coordinates in spatial and Fourier domains, respectively. We also use $f(\boldsymbol{x})$ and $f_{\mathrm{pol}}(r, \theta)$ for the Cartesian and polar parametrizations of the same function $f$ (similarly in the Fourier domain).

Definition 1 A function $\psi$ on the plane is steerable in the finite basis $\left\{\xi^{(1)}, \ldots, \xi^{(N)}\right\}$ if for any rotation matrix $\mathbf{R}_{\theta_{0}}$, we can find coefficients $u_{1}\left(\theta_{0}\right), \ldots, u_{N}\left(\theta_{0}\right)$ such that

$$
\psi\left(\mathbf{R}_{\theta_{0}} \boldsymbol{x}\right)=\sum_{n=1}^{N} u_{n}\left(\theta_{0}\right) \xi^{(n)}(\boldsymbol{x}) .
$$

In particular, if

$$
\psi_{\mathrm{pol}}(r, \theta)=\sum_{n \in S} u_{n} \xi_{\mathrm{pol}}^{(n)}(r, \theta)
$$

for some finite set $S \subset \mathbb{Z}$, where $\xi^{(n)}$ is polar-separable as

$$
\xi_{\mathrm{pol}}^{(n)}(r, \theta)=\eta_{n}(r) \mathrm{e}^{\mathrm{j} n \theta}
$$

then

$$
\psi_{\mathrm{pol}}\left(r, \theta+\theta_{0}\right)=\sum_{n} \mathrm{e}^{\mathrm{j} n \theta_{0}} u_{n} \xi_{\mathrm{pol}}^{(n)}(r, \theta) .
$$

This means that any function $\psi$ of the above form is steerable, and its steered coefficients for angle $\theta_{0}$ are obtained from the original ones by multiplication with $\mathrm{e}^{\mathrm{j} n \theta_{0}}$. 


\subsection{Steerable wavelet frames}

We now briefly review a construction that combines the concept of steerability with the concept of a tight wavelet frame (we refer to [8] for more details). The resulting transform analyzes images in a mathematically stable way using multiple dilated versions of a set of steerable templates. Hence it can be used for detecting features of interest at different scales and for extracting their local orientation.

The starting point is an isotropic function $h(\rho)$ such that the shifts and dilations of $\mathscr{F}^{-1}\{h(\rho)\}$ form a wavelet frame. In this work we use Simoncelli's isotropic wavelet [9] defined by

$$
h(\rho)=\left\{\begin{array}{cl}
\cos \left(\frac{\pi}{2} \log _{2}\left(\frac{2 \rho}{\pi}\right)\right), & \frac{\pi}{4}<\rho \leq \pi \\
0, & \text { otherwise }
\end{array}\right.
$$

One can then show that the inverse Fourier transform of

$$
\hat{\xi}_{\mathrm{pol}}^{(n)}(\rho, \phi)=h(\rho) \mathrm{e}^{\mathrm{j} n \phi} .
$$

is of the form (2) and is therefore steerable. In addition, $\xi_{\mathrm{pol}}^{(n)}(\rho, \phi)$ still generates a tight frame. By choosing $N$ distinct values for the integer $n$ we can thus form an $N$-channel tight frame of steerable wavelets.

In the sequel we will use $i$ and $\boldsymbol{k}$ to index the scale and shift of the basis functions. The values of $n$ are taken from a predefined set $S=\left\{n_{1}, \ldots, n_{N}\right\}$, called the set of harmonics, which is one of our design parameters.

From the original steerable wavelet representation given above, we can construct new steerable representations, by using an orthogonal shaping matrix $\mathbf{U}$ to define the new steerable frame functions:

$$
\left[\begin{array}{c}
\psi_{i, \boldsymbol{k}}^{(1)} \\
\vdots \\
\psi_{i, \boldsymbol{k}}^{(N)}
\end{array}\right]=\mathbf{U}\left[\begin{array}{c}
\xi_{i, \mathbf{k}}^{\left(n_{1}\right)} \\
\vdots \\
\xi_{i, \boldsymbol{k}}^{\left(n_{N}\right)}
\end{array}\right] .
$$

The number of channels here corresponds to the number of harmonics.

The new wavelets span the same space as the wavelet frame $\xi_{i, k}^{(n)}$. The role of the shaping matrix $\mathbf{U}$ is to give the wavelet functions $\psi_{i, k}^{(n)}$ a desired angular profile.

Our goal is to design a wavelet for the detection of symmetric patterns by optimizing a coefficient vector $\boldsymbol{u}=\left(u_{1}, \ldots, u_{N}\right)$, with $\boldsymbol{u}^{\mathrm{H}} \boldsymbol{u}=1$. This vector forms the first row of $\mathbf{U}$. From this vector we get the first channel of wavelets $\psi=\sum_{n} u_{n} \xi_{n}$ and find the remaining channels by choosing $N-1$ other vectors orthogonal to $\boldsymbol{u}$ (note that these vectors can be complex). Other non-orthogonal designs may also be considered if specific design objectives are to be achieved, but these fall outside the focus of the present paper.

\section{DESIGN OF M-FOLD SYMMETRIC WAVELETS}

We impose an M-fold symmetric pattern on $\psi$ by minimizing an energy functional that favors $\mathrm{M}$-fold symmetries. Since rotation and symmetries in the space domain carry over to the frequency domain we can define this energy in the latter. This results in a substantial simplification of the problem, owing to the fact that the wavelet function $(\psi)$ is polar-separable in the Fourier domain but not in space. As a result, we can optimize the angular profile of the wavelet by defining the Fourier-based energy for the angular factor alone, without any influence from the radial factor.

Using (3) and (1), we arrive at the following polar factorization of $\hat{\psi}$ :

$$
\hat{\psi}(\rho, \phi)=h(\rho) \sum_{n \in S} u_{n} \mathrm{e}^{\mathrm{j} n \phi}=h(\rho) \hat{\psi}(\phi),
$$

where, with some abuse of notation, we have used $\hat{\psi}(\phi)$ to denote the angular factor of $\hat{\psi}(\rho, \phi)$.

We then define the energy functional $E[\hat{\psi}]$ as

$$
E[\hat{\psi}]=\int_{-\pi}^{+\pi}|\hat{\psi}(\phi)|^{2} w(\phi) \mathrm{d} \phi,
$$

where $w(\phi) \geq 0$ is an $M$-fold symmetric weighting function with $M$ equidistant minima. This will force the solution $\hat{\psi}$ to be localized symmetrically near the M-fold minima.

It follows from (4) that the above integral energy can be expanded as

$$
\begin{aligned}
E[\hat{\psi}] & =\int_{-\pi}^{+\pi}\left|\sum_{n \in S} u_{n} \mathrm{e}^{\mathrm{j} n \phi}\right|^{2} w(\phi) \mathrm{d} \phi \\
& =\sum_{n^{\prime} \in S} \sum_{n \in S} \overline{u_{n}^{\prime}} u_{n} \int_{-\pi}^{+\pi} \mathrm{e}^{\mathrm{j}\left(n-n^{\prime}\right) \phi} w(\phi) \mathrm{d} \phi \\
& =\sum_{n^{\prime} \in S} \sum_{n \in S} \overline{u_{n}^{\prime}} W\left(n-n^{\prime}\right) u_{n}=\boldsymbol{u}^{\mathrm{H}} \mathbf{W} \boldsymbol{u},
\end{aligned}
$$

where $\mathbf{W}$ is the Hermitian-symmetric matrix with entries indexed by $n, n^{\prime} \in S$ :

$$
[\mathbf{W}]_{n, n^{\prime}}=\int_{-\pi}^{+\pi} \mathrm{e}^{\mathrm{j}\left(n-n^{\prime}\right) \phi} w(\phi) \mathrm{d} \phi .
$$

Based on the previous considerations we have a quadratic optimization problem with quadratic constraints. Such problems are in general difficult to solve, but this particular instance (with $\boldsymbol{u}^{\mathrm{H}} \boldsymbol{u}=1$ ) admits an analytic solution that is readily computable: the solution is simply an eigenvector corresponding to the smallest eigenvalue of $\mathbf{W}$.

A particular example of a 3-fold symmetric wavelet obtained by the proposed method can be seen in Fig. 1. The weight function $w(\phi)$ is $\frac{2 \pi}{M}$-periodic with $w(\phi)=\phi^{2}$ for $|\phi| \leq \frac{\pi}{M}$, to achieve maximal energy concentration about the minima in the sense of variance.
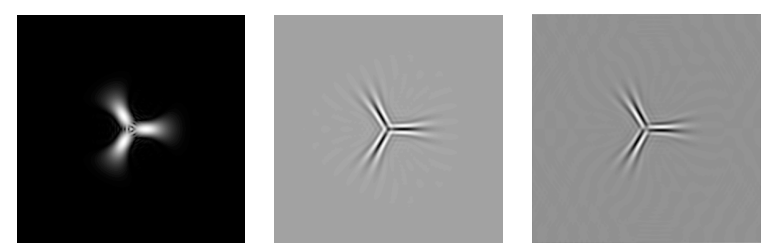

Fig. 1: 3-fold symmetric wavelet in the space domain; from left: magnitude, real and imaginary parts. Harmonics: $S=\{3 n: n=$ $0, \ldots, 9\}$. 


\section{APPLICATION TO JUNCTION DETECTION}

\subsection{Steering}

To detect symmetry centers in an image we need to determine the maximum response over all orientations of the detector wavelet. The task of finding the optimal angle is also known as "steering" and can be formulated as follows. Let $\left\{q_{1}, \ldots, q_{N}\right\}$ be the coefficients of the $N$ channels that are computed by analyzing the image at scale $i$ and position $k$ using the original $N$ channel wavelets: $\left\{\xi_{i, k}^{\left(n_{1}\right)}(r, \theta), \ldots, \xi_{i, k}^{\left(n_{N}\right)}(r, \theta)\right\}$. We wish to rotate the wavelets $\psi$ at each $(i, \boldsymbol{k})$ to maximize $\left|\left\langle\psi_{i, k}\left(\cdot, \cdot+\theta_{0}\right), f\right\rangle\right|$ as a function of $\theta_{0}$.

Thus, the function to maximize at each $(i, \boldsymbol{k})$ is

$$
Q\left(\theta_{0}\right)=\left|\left\langle\sum_{n \in S} \mathrm{e}^{\mathrm{j} n \theta_{0}} u_{n} \xi_{i, k}^{(n)}, f\right\rangle\right|=\left|\sum_{n \in S} q_{n} \overline{u_{n}} \mathrm{e}^{-\mathrm{j} n \theta_{0}}\right|^{2}
$$

(note that the inner product is conjugate-linear in the first argument).

This is a trigonometric polynomial in $\theta_{0}$ which can be maximized efficiently by finding the roots of its derivative.

More precisely, we can use the fact that for an $M$-fold pattern we use harmonics that are multiples of $M$, in order to reduce the order of the polynomial to solve. This is done by making a change of variable in the following way:

For a set of harmonics $S=\left\{M k: k=k_{0}, k_{0}+1, \ldots, k_{0}+N-1 \in\right.$ $\mathbb{Z}\}$, we introduce the variable $z=\mathrm{e}^{\mathrm{j} M \theta_{0}}$. We are then looking for the maximum of the polynomial

$$
Q(z)=\left(\sum_{k=k_{0}}^{k_{0}+N-1} r_{k} z^{k}\right) \overline{\left(\sum_{k=k_{0}}^{k_{0}+N-1} r_{k} z^{k}\right)}
$$

on the unit circle, where $r_{k}=q_{M k} \overline{u_{M k}}$. This maximum happens at a point where the derivative of $Q(z)$ with respect to $\theta_{0}$ is zero. We find all such points and evaluate $Q(z)$ at all of them to find the maximum.

$Q(z)$ can be transformed to an easily processable form:

$$
Q(z)=\sum_{k} s_{k} z^{k}
$$

where $s=r[\cdot] * \bar{r}[-\cdot]$ is the auto correlation of $r$. Then

$$
\frac{\mathrm{d}}{\mathrm{d} \theta_{0}} Q(z)=(\mathrm{j} M) \sum_{k} k s_{k} z^{k} .
$$

Having the coefficients of $\frac{\mathrm{d}}{\mathrm{d} \theta_{0}} Q(z)$, we can compute its complex roots. Let $\zeta$ be the root on the unit circle for which $Q(z)$ is maximized. From the definition $z=\mathrm{e}^{\mathrm{j} M \theta_{0}}$, the optimal steering angle is then given by the relation

$$
\theta_{0}=\frac{\measuredangle \zeta}{M}
$$

Note that due to $M$-fold symmetry, $\theta_{0}+m \frac{2 \pi}{M}, m \in \mathbb{Z}$, are equivalent solutions.

\subsection{Summary of the algorithm}

The algorithm we propose estimates the number of junctions (without a priori knowledge), their center and the orientation of the corresponding edges. The main steps of the algorithm are:
(1) (Wavelet analysis with optimally steered wavelets) First we design the M-fold symmetric wavelet in the Fourier domain based on the previously described method. We decompose the image with the steerable wavelet and, at each point, we determine the angle $\theta_{0}$, such that the "detector response" (7) is maximized. The output of this first stage is a map of maximal steerable wavelet responses $(Q(i, k))$ at every scale and position. (2) (Maximum-intensity projection across scales)

To aggregate the junction detections from the different scales we perform a maximum-intensity projection. For each position we keep the coefficient corresponding to the largest detector response:

$$
Q(\boldsymbol{k})=\max _{i}(Q(i, \boldsymbol{k}))
$$

This is justified since the wavelets are normalized.

(3) (Thresholding and local maxima detection)

Assuming that there are many more ordinary pixels in the image than junctions we can reduce the amount of computations and neglect the background pixels by suppressing the points having a smaller detector response than the mean of the whole image.

Since the same symmetry center (junction) may be detected at several nearby locations and across scales, to have a better localization of junctions and also eliminate redundant detections, we follow the thresholding step by a local maximum search (within a window of predefined size).

\section{EXPERIMENTAL RESULTS}

To evaluate the performance of the algorithm, we first use a test image (Fig. 2) consisting of a 3-4 uniform tiling [10], where the goal is to detect 3 -fold symmetric junctions.

We study the effect of the following parameters on the performance of the algorithm: (i) the size of the junctions, (ii) the blurring of the edges, (iii) and the presence of ambient noise. The first image is the original one, here one can observe the detections at different scales. The second image is the blurred version of the original one with a $3 \times 3$ moving average kernel. The third image is corrupted with additive Gaussian noise with a mean of zero and standard deviation of 50 (for an intensity range of 0 to 255). To the last image we add salt and pepper noise, by randomly replacing $5 \%$ of the pixels with black and 5\% with white pixels.

Figs. 3 and 4 show the results of our detection algorithm applied to real microscopy images. Fig. 3 shows a stem cross section of Convallaria, cropped to size $1024 \times 1024$ pixels. Fig. 4 shows a leaf cross section of Maize, cropped to size $1024 \times 1024$ pixels. In both cases most of the junctions are detected, including highly distorted and noisy ones.

\section{CONCLUSION}

We presented a construction of $M$-fold symmetric steerable tight wavelet frames and an algorithm for the detection of symmetries and junctions in microscopy images. The attractive features of the algorithm are (i) the multiscale approach and the angular selectivity, which make it possible to obtain a precise estimate of the junctions across scales; (ii) the steering property of the detector wavelet, which allows us to make the detections very efficiently regardless of the orientation of the pattern, without a need to reprocess the image for each angle; and (iii) the tight frame property that permits energy-based analysis and reconstruction from features. Future work can focus on classification of junctions based on their symmetry order where we can take advantage of 

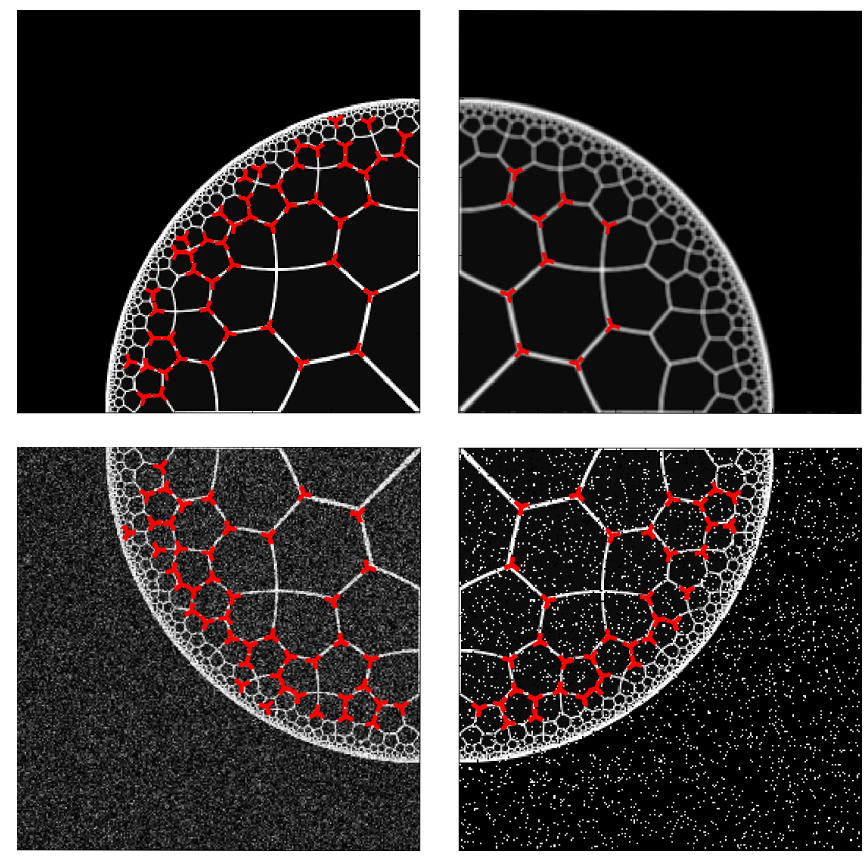

Fig. 2: Detection of 3-fold symmetric junctions in synthetic data. Row 1, Col. 1: Junctions of different size; Row 1, Col. 2: Smoothed junctions of different size; Row 2, Col. 1: Junctions of different size corrupted with additive Gaussian noise, mean: 0, standard deviation: 50; Row 2, Col. 2: Junctions of different size corrupted with "Salt and Pepper" noise, 5\%.

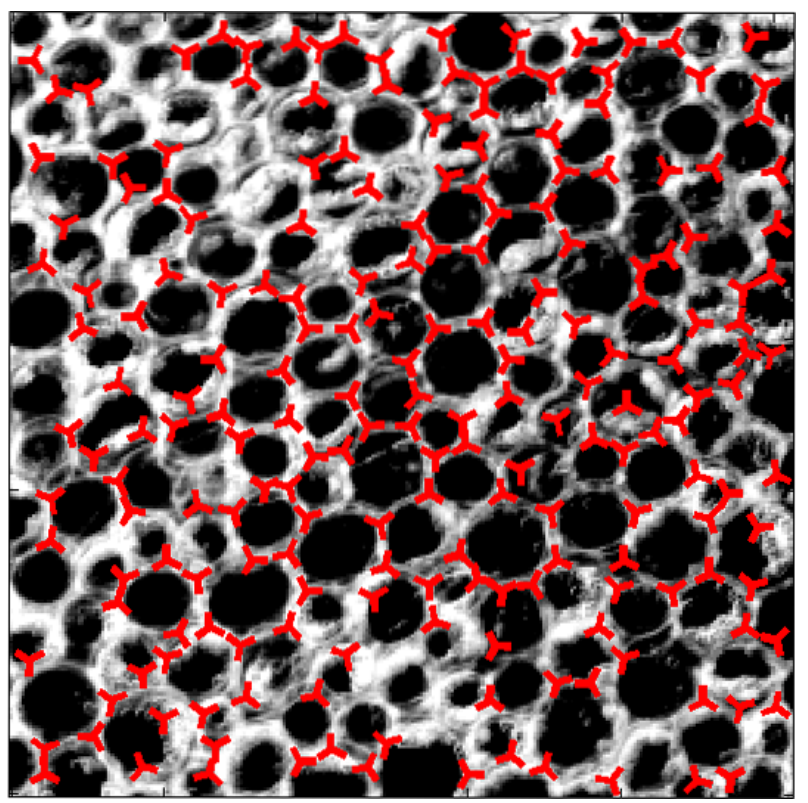

Fig. 3: Junctions (shown using red stars) in a Convallaria stem cross section (Courtesy of J. Artacho, BIOP, EPFL).

the selectivity of the approach, and on benefiting from the tight frame property for the enhancement and reconstruction of images based on symmetric features.

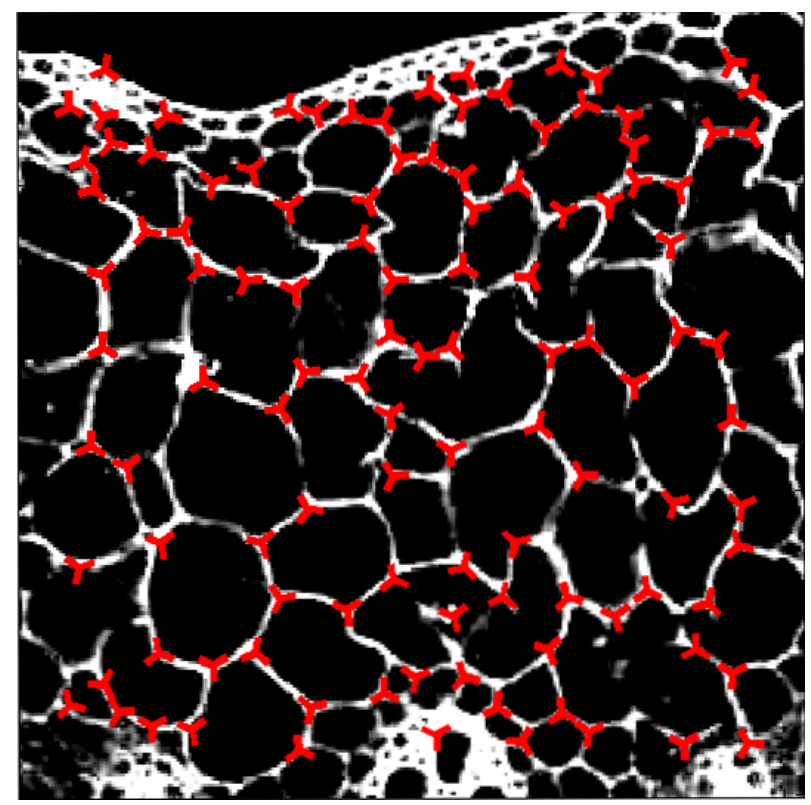

Fig. 4: Junctions in the cross-section through the middle of a Maize leaf [11].

\section{ACKNOWLEDGEMENTS}

This work was supported by the ERC (ERC-2010-AdG 267439-FUN-SP), the Hasler Foundation and the Indo-Swiss Joint Research Program.

\section{REFERENCES}

[1] D. J. Beymer, "Finding junctions using the image gradient," A.I. Memos, M.I.T. Artifical Intelligence Laboratory, vol. AIM-1266, pp. 1-46, Dec. 1991.

[2] U. Köthe, "Edge and junction detection with an improved structure tensor," in Pattern Recognition, ser. Lecture Notes in Computer Science, B. Michaelis and G. Krell, Eds. Springer Berlin / Heidelberg, 2003, vol. 2781, pp. 25-32.

[3] L. Parida, D. Geiger, and R. Hummel, "Junctions: detection, classification, and reconstruction," IEEE Trans. Pattern Analysis Mach. Intell., vol. 20, no. 7, pp. 687-698, July 1998.

[4] M. Jacob and M. Unser, "Design of steerable filters for feature detection using Canny-like criteria," IEEE Trans. Pattern Analysis Mach. Intell., vol. 26, no. 8, pp. 1007-1019, August 2004.

[5] M. Mühlich, D. Friedrich, and T. Aach, "Design and implementation of multi-steerable matched filters," IEEE Trans. Pattern Analysis Mach. Intell., vol. 34, no. 2, pp. 279-291, 2012.

[6] D. Slepian, "Prolate spheroidal wave-functions, Fourier-analysis, and uncertainty - V: The discrete case," Bell System Tech. J., vol. 57, pp. 1371-1430, 1978.

[7] W. Freeman and E. Adelson, "The design and use of steerable filters," IEEE Trans. Pattern Analysis Mach. Intell., vol. 13, no. 9, pp. 891 -906, sep 1991.

[8] M. Unser and N. Chenouard, "A unifying parametric framework for 2D steerable wavelet transforms," SIAM J. Imaging Sci., in press.

[9] J. Portilla and E. P. Simoncelli, "A parametric texture model based on joint statistics of complex wavelet coefficients," Int. J. Comput. Vision, vol. 40, no. 1, pp. 49-70, Oct. 2000.

[10] Uniform tilings in hyperbolic plane. [Online]. Available: http: //en.wikipedia.org/wiki/Uniform_tilings_in_hyperbolic_plane

[11] Cross-section through the central part of a leaf of corn. [Online]. Available: http://www.vcbio.sci.kun.nl/en/image-gallery/show/ PL0184/ 